\title{
MODULATION OF METHOTREXATE EFFICACY BY GREEN TEA \\ POLYPHENOLS IN RAT ADJUVANT ARTHRITIS
}

PONIST $^{1 *}$ Silvester, GARDI ${ }^{2 *}$ Concetta, PASKOVA ${ }^{3}$ Ludmila, SVIK $^{1}$ Karol, SLOVAK ${ }^{1}$

Lukas, BILKA ${ }^{3}$ Frantisek, TEDESCO ${ }^{4}$ Idolo, BAUEROVA ${ }^{1 \S}$ Katarina, RUSSO ${ }^{4 \S}$ Gian Luigi

${ }^{1}$ Centre of Experimental Medicine, Slovak Academy of Sciences, Bratislava, Slovakia;

${ }^{2}$ Department of Molecular and Developmental Medicine, University of Siena, Siena, Italy;

${ }^{3}$ Department of Cell and Molecular Biology of Drugs, Faculty of Pharmacy, Comenius University, 83232 Bratislava, Slovakia; ${ }^{4}$ National Research Council, Institute of Food Sciences, Avellino, Italy

\section{*Equal contribution}

\section{§To whom correspondence should be addressed:}

Gian Luigi Russo National Research Council, Institute of Food Sciences

Via Roma, 64

83100 - Avellino - Italy

Phone (office): +39 0825299331 - Phone (mobile): +39 3299064414

E-mail: glrusso@isa.cnr.it

PharmDr. Katarína Bauerová, PhD., DrSc.

Centre of Experimental Medicine SAS

Slovak Academy of Sciences

Dúbravská cesta 9, 84104 Bratislava - Slovak Republic

Phone: +421-2-32295731

E-mail: katarina.bauerova@savba.sk 


\section{Highlights}

- Co-administration of green tea extract and methotrexate (MTX) has been studied in arthritic rats

- A green tea extract gradually decreased the effectiveness of MTX on arthritic score - A green tea extract alone and with MTX reduced MCP-1, but counteracted IL-17 only at day 14

- A green tea extract inhibited iNOS mRNA and GGT, but in a less extent compared to MTX - Long-term administration of green tea extract may inhibit the therapeutic action of methotrexate 


\begin{abstract}
Methotrexate is a widely used drug for treatment of rheumatoid arthritis. The present study aimed to investigate if tea polyphenols administered as decaffeinated extract $\left(\right.$ GreenSelect ${ }^{\circledR}$, GS) can ameliorate methotrexate treatment of rat adjuvant arthritis. The study design lasted 28 days and included healthy animals administered with GS in the daily dose of $200 \mathrm{mg} / \mathrm{kg}$, arthritic rats administered or not with GS, arthritic animals administered with methotrexate in single treatment and in combination with GS. Arthritic score and changes in body weigh were measured during the treatment while inflammatory markers (monocyte chemotactic protein-1, IL-17 and inducible NO-synthase mRNA) and biochemical parameters (activity of gammaglutamyltransferase and expression of heme oxygenase-1) at the end of the treatment. The association between GS and methotrexate was less efficient in ameliorating the arthritic score compared to methotrexate alone and, in general, GS did not improve the inflammatory and biochemical markers except for monocyte chemotactic protein-1. GS did not increase the plasma antioxidant capacity, suggesting a pro-oxidant effect and worsened the body weight loss. The results suggest that long-term administration of GS may inhibit the therapeutic action of methotrexate in arthritic rats due to the inhibitory activity of (-)-epigallocatechin-3gallate on folate transporter.
\end{abstract}

Key words: arthritis, green tea polyphenols, methotrexate, inflammation, antioxidants 


\section{Introduction}

Rheumatoid arthritis (RA) is a common severe joint disease-affecting people of all age. To control inflammatory symptoms and pain, disease modifying antirheumatic drugs are commonly used although they are associated with undesirable side effects. Many clinical studies show that combination therapy is usually more effective than a single treatment (monotherapy) in order to control inflammation in RA. Methotrexate (MTX), a folic acid antagonist, became the predominant immunosuppressive agent used in the treatment of patients with RA [1].

Green tea extract (GTE) is widely used in different dietary supplements for its proved health benefits. GTE restored the oxidative state of the liver and brain of adjuvant-induced arthritic rats, a model for human RA [2]. The treatment produces significant reduction in protein and lipid damage in liver, brain and plasma. It also reduces the tissue reactive oxygen species (ROS) content and increases the antioxidant capacity of the plasma. The antioxidant defences, which are diminished by arthritis, were improved by GTE treatment, as revealed by the restoration of the glutathione and protein thiol levels [3]]. Several factors including inflammation and oxidative stress are believed to play a role in the development of chronic joint diseases. There are significant evidence that (-)-epigallocatechin-3-gallate (EGCG), the predominant green tea flavonoid in GTE, negatively modulates signal transduction pathways that play important roles in inflammation and joint damage in arthritis [4]. In details, EGCG inhibits advance glycation end products (AGEs), induces expression of MMP-13 (matrix metalloproteinases-13) in human osteoarthritis chondrocytes [ $[\underline{5}]$ and induces the production of MMP-1 and MMP-3 in RA synovial fibroblasts [ㅁ] .

Besides their anti-inflammatory and oxidative stress reducing activities, GTE and EGCG are able to reduce the transport of folic acid and MTX into the cell. The proton coupled folate transporter (PCFT)-mediated folate uptake is competitively inhibited by EGCG. The uptake 
of the PCFT substrate MTX is also competitively inhibited by EGCG. Authors of this study suggested that, when folate or MTX are ingested with tea, it is likely that the intestinal absorption of these compounds by PCFT is inhibited, which could result in insufficient efficacy of drug MTX []].

The present study aims to demonstrate if a standardized preparation of decaffeinated extract of green tea (Greenselect ${ }^{\circledR}$, GS) can modulate MTX treatment in a rat model of adjuvant arthritis and improve the efficacy of MTX therapy.

\section{Methods}

\subsection{Animal study}

Male Lewis rats were obtained from the Breeding Farm Dobra Voda (Slovakia) and housed five per cage under standard conditions with food and water ad libitum and a $12 \mathrm{~h} \mathrm{light} / 12 \mathrm{~h}$ dark cycle. The experimental protocol was approved by the Ethics Committee of Centre of Experimental Medicine of The Slovak Academy of Sciences (authorization n. 3144/16-221/3) and by the Slovak State Veterinary and Food Administration in accordance with the European Convention for the Protection of Vertebrate Animals Used for Experimental and Other Scientific Purposes. The study was in accordance with Slovak legislation.

\subsubsection{Induction of Adjuvant Arthritis}

Adjuvant arthritis (AA) was induced to male Lewis rats weighing 160-180 $\mathrm{g}$ by a single intradermal injection of $0.1 \mathrm{~mL}$ suspension of heat-inactivated Mycobacterium butyricum (Difco Laboratories, Detroit, MI, USA) in incomplete Freund's adjuvant at the base of the tail as previously reported []].

\subsubsection{Experimental Design and Treatments}


Rats were randomized into six groups. The experimental design included: healthy animals (HC); HC administered with GS (kindly provided by Indena, Italy; data on Greenselect ${ }^{\circledR}$ composition can be retrieved from https://www.indena.com) in an oral daily dose of 200 mg/kg b.w. (HC-GS); arthritic-induced animals (AA); AA rats administered with GS in oral daily dose of $200 \mathrm{mg} / \mathrm{kg}$ b.w. (AA-GS); AA rats administered with MTX in oral daily dose of $0.3 \mathrm{mg} / \mathrm{kg}$ b.w. twice a week (AA-M); AA rats administered with a combination of GS and MTX (GS+MTX). All treatments lasted 28 days and included eight animals per group. GS and MTX were administered by oral gavage on day 14, blood samples were taken from eye

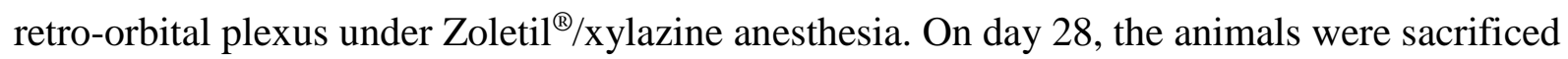
under the same anaesthesia and blood for plasma preparation was withdrawn from each rat along with the different tissues (liver, spleen, joint and lung) employed for the subsequent analyses. All samples were stored at $-80^{\circ} \mathrm{C}$ until biochemical analyses. The selected oral dose of GS was considered to be safe based on previous toxicity studies $[\underline{9}, \underline{10}]$.

\subsubsection{Change of body weight}

Change of body weight (g) was measured on days 1, 14, 21 and 28 and calculated as the difference of the body mass determined on days 14,21 and 28 and the body weight measured at the beginning of the experiment (day 1).

\subsubsection{Arthritic score}

The arthritic score was measured as the total score of hind paw volume (mL; max. points 8) plus paw diameter of forelimb (mm, max. points 5) plus diameter of scab in the site of $M$. butyricum application, measured in parallel with the spinal column (mm, max. points 5) for each animal [11]. 


\subsection{In vitro assays}

\subsubsection{Markers of inflammation and total antioxidant status in plasma}

Plasma interleukin-17 (IL-17) and monocyte chemotactic protein-1 (MCP-1) levels were measured as inflammatory markers using ELISA kits from R\&D Systems Quantikine ${ }^{\circledR}$ (Minneapolis, MN, USA) and eBioscience ${ }^{\circledR}$ (Waltham, MA, USA), respectively. The assay procedures were as reported by the manufacturers' instructions.

The total antioxidant capacity was measured in the plasma of rats treated as indicated above employing the ABTS (2,20-azinobis(3-ethylbenz-thiazoline-6-sulfonic acid)) (Sigma-Aldrich, Milan, Italy) radical cation decolorization, essentially as previously reported [12] with few modifications. Briefly, $5 \mu \mathrm{L}$ of plasma were added to $200 \mu \mathrm{L}$ of acetate buffer $\mathrm{pH} 5.8$ before the addition of $5 \mu \mathrm{L}$ of ABTS radical solution. After 5 min of incubation at room temperature, absorbance at $660 \mathrm{~nm}$ was measured and values expressed as micromolar equivalent of ascorbic acid, an antioxidant present in the plasma [12].

\subsubsection{Relative mRNA expression by quantitative RT-PCR}

Relative mRNA expressions of iNOS (inducible nitric oxide synthase) was analysed by qPCR. Total RNA was extracted from the livers of rats treated as reported above at day 28 using RNAzol RT (Sigma-Aldrich) and reverse transcribed using the PrimeScript RT Reagent Kit (Takara) following the manufacturer's guidelines. Amplification and detection of cDNA of target and reference genes were performed in triplicates using HOT FIREPol EvaGreenR qPCR Mix Plus (ROX) (Solis Biodyne) on a 7300 Real-Time PCR System (Applied Biosystems). Primer sequences were: iNOS Fw:5'-AAAACCCCAGGTGCTATTCCC-3’, iNOS Rev: 5'-GTGGTGAAGGGTGTCGTGAA-3', $\beta$-actin Fw: 5'TCAAGATCATTGCTCCTCCTG-3', $\beta$-actin Rev: 5'-AGGGTGTAAAACGCAGCTCA-3'. Relative mRNA expressions of iNOS was analysed using the $\Delta \Delta \mathrm{Ct}$ value method. $\beta$-Actin 
was used as a reference gene. PCR products were evaluated by melting curve analysis to confirm the specific amplification. The sequences of the primers were designed and checked using Primer3 and Oligo Analyzer 1.0.3.

\subsubsection{Immunoblot analysis of heme oxygenase-1 (HO-1)}

Protein samples from joint and lung homogenates were resolved on a 10\% SDS-

polyacrylamide gel and transferred onto nitrocellulose membrane (Whatman GmbH, Dassel, Germany), as previously reported [12]. Immunoblots were performed using primary antibodies for HO-1 (1:1000; Abcam, Cambridge, UK) and $\beta$-actin (Thermo Fisher Scientific, Monza, Italy) was used as loading control. Immunodetected proteins were visualized using ECL kit (BioRad, Hercules, CA, USA).

\subsubsection{Activity of cellular $\gamma$-glutamyltransferase in spleen tissue}

The activity of cellular $\gamma$-glutamyltransferase (GGT) in spleen tissue homogenates was measured as previously reported [13]. Samples were homogenized in a buffer $(2.6 \mathrm{mM}$ $\mathrm{NaH}_{2} \mathrm{PO}_{4}, 50 \mathrm{mM} \mathrm{Na} \mathrm{HPO}_{4}, 15 \mathrm{mM}$ EDTA, and $\left.68 \mathrm{mM} \mathrm{NaCl} ; \mathrm{pH} 8.1\right)$ at 1:9 (w/v) by UltraTurax TP 18/10 (Janke \& Kunkel, Germany) for 1 min at $0^{\circ} \mathrm{C}$. Substrates $(8.7 \mathrm{mM} \gamma$ glutamyl-p-nitroanilide, $44 \mathrm{mM}$ methionine) were added to $65 \%$ isopropyl alcohol to final concentrations of $2.5 \mathrm{mM}$ and $12.6 \mathrm{mM}$, respectively. After incubation for $60 \mathrm{~min}$ at $37^{\circ} \mathrm{C}$, the reaction was stopped with $2.3 \mathrm{~mL}$ of cold methanol and the tubes were centrifuged for $20 \mathrm{~min}$ at $5000 \mathrm{rpm}$. Absorbance of supernatant was measured in a Specord 40 spectrophotometer (Analytic Jena AG) in a $0.5 \mathrm{~cm}$ cuvette at $406 \mathrm{~nm}$. Reaction mixtures in the absence of either substrate or acceptor were used as reference samples.

\subsection{Statistical analysis}


Mean and S.E.M. values were calculated for each parameter in each group (seven to eight animals/group). Statistically significant differences among treated, untreated, and control groups were tested using parametric Analysis of Variance (ANOVA). Post hoc tests (TukeyKramer (ANOVA)) were applied in situations where differences among groups were significant at the level of significance $\alpha=0.05$. After post hoc testing, the following significance levels were specified: extremely significant $(P<0.001)$, highly significant $(P<$ $0.01)$, significant $(P<0.05)$, and not significant $(P>0.05)$. Data reported for the AA group were compared to those measured in the HC group (*); data from the AA-M and AA-GS-M groups were compared with those measured in the AA group (+ and \#, respectively).

\section{Results}

\subsection{Effects of GS, MTX and their combination on body mass and arthritic score}

During the AA development, body weight of untreated AA animals significantly decreased, as expected, from day 14 to day 28 (Fig. 1). The GS treatment in control rats showed a trend of body weight reduction, although not significant. Differently than GS, MTX rescued significantly the loss of weight in the AA group on days 21 and 28. Unexpectedly, at the same time points, the addition of GS to MTX treatment abolished the positive effect on rat body weight caused by MTX in the AA group (Fig. 1).

The arthritic score showed the expected increase in the AA group during the entire duration of the treatment which was not influenced by the treatment with GS. MTX and GS+MTX significantly ameliorated this parameter, although the combined treatment was less efficient in decreasing the arthritic score points respect to MTX single treatment (Fig. 2).

\subsection{Effect of GS, MTX and their combination on inflammatory markers}


The induction of AA significantly increased the plasma levels of MCP-1 on days 14 and 28. On day 14, the administration of both GS and MTX in monotherapy was without significant effects, but their combination significantly decreased the level of MCP-1 when compared to the AA and AA-MTX groups (Fig. 3A). On day 28, administration of GS, MTX and their association significantly reduced the MCP-1 levels when compared to untreated AA animals. Moreover, combination of GS+MTX was more effective than MTX single treatment in reducing plasma MCP-1 concentration (Fig. 3A).

On day 14, IL-17 concentration followed the same increasing trend than MCP-1 in the AA group. The administration of GS indicated a potential beneficial effect, although not significant, while MTX in monotreatment and in combination with GS significantly decreased the plasma levels of IL-17 when compared to the AA group (Fig. 3B). However, this effect was clearly due to MTX rather than to its association with GS. On day 28, the concentration of IL-17 strongly decreased (about 3-fold) compared to the previous time-point and the treatment with MTX and/or GS was ineffective (Fig. 3B).

\subsection{Effect of GS, MTX and their combination on biochemical markers}

\subsubsection{Expression of iNOS $m R N A$}

iNOS is expressed in response to pro-inflammatory stimuli and produces NO as a defence response. The associated overexpression of iNOS mRNA has been described in several pathophysiological conditions, including rheumatoid arthritis [14]. Therefore, we verify the capacity of GS, in association with MTX, to modulate the levels of iNOS mRNA in our experimental model, since we previously measured iNOS increase in liver of 28 days AA rats [15]. Administration of GS, MTX and their combination significantly reduced the expression of iNOS mRNA when compared to the AA group of animals (Fig. 4A), but also in this case, the efficacy of GS in the AA-GS-M group was negligible. 


\subsubsection{GGT activity}

GGT is localized in several human tissues and its increase has been detected in active immune cells, hypothesizing an involvement of GGT in the immune responses. Since lymphocytes accumulate in the RA synovium, GGT can be implicated in inflamed synovium and arthritisrelated osteolysis. In fact, expression of GG has been detected in joints of patients with RA and in animal model of collagen-induced arthritis [16]. Based on these evidence, GGT activity in the spleens of AA rats resulted about 3-fold higher compared to HC group (Fig. 5). Administration of GS, MTX and their combination significantly decreased the enzymatic activity compared to the AA group with the MTX treatment that resulted the most effective, independently from the presence of GS (Fig. 4B).

\subsubsection{Expression of $\mathrm{HO}-1$}

Given that HO-1 shows antioxidant properties and is a key therapeutic target of inhibition of inflammation, we investigated the effect of GTE on HO-1 protein levels in lung and joint of AA rats. As depicted in Fig. 4C, in the AA group, the expression of HO-1 protein in lung tissue was significantly decreased, suggesting a lowering of antioxidant defences. Only MTX in monotherapy significantly increased the expression of HO-1 to control value. Neither GS nor combination with MTX treatment were able to affect the decreased expression of HO-1 in the AA group. Expression of HO-1 in joint tissue was unaffected by AA and administration of substances studied (data not shown). Thus, GS failed to exert a protective effect in both lung and join from AA animals.

\subsection{GS effect on plasma antioxidant power}


We measured the antioxidant capacity in plasma of AA rats following GS and MTX treatments using the colorimetric ABTS assay. As reported in Fig. 4D, GS decreased the plasma antioxidant power in control group (HC-GS) of about 20\%, suggesting its potential and unpredicted pro-oxidant activity. A comparable decrease (33\%) was measured in the AA group, as expected. The treatment with MTX further decreased the plasma antioxidant power in AA rats, lowering it of about $25 \%$ (comparing AA vs AA-M groups). The treatment with GS in AA and AA-M groups failed to ameliorate the antioxidant response. In fact, in the former (AA-GS group), no significant improvement compared to AA rats was obtained in the plasma antioxidant power by GS treatment. Similarly, the slight increase in the antioxidant power observed in AA-GS-M group respect to the AA-M one was statistically not significant and, in any case, remained significantly lower if compared to the AA group.

Overall, these data suggest that GS failed to behave as an antioxidant mixture, ameliorating the antioxidant capacity in the plasma of arthritic rats and counteracting the oxidative stress associated to AA induction. On the opposite, and unexpectedly, healthy animals treated with GS showed a lower antioxidant capacity compared to controls (HC) confirming the prooxidant trend of GS treatment (Fig. 4D).

\section{Discussion}

Various disease-modifying anti-rheumatic drugs (DMARDs) were developed in the last years for the treatment of RA [17]. Biological treatments, such as etanercept and infliximab, are a newer form of treatment for RA, but the high cost and the need of repeated injections inhibited their widespread use. Thus, conventional DMARDs such as MTX have been continued to be used in clinical setting $[\underline{18}, \underline{19}]$. MTX became the most frequently used DMARD in the therapy of RA, but its administration is limited being sometimes ineffective and for the presence of manifold adverse effects [20-22]. 
In this scenario, alternative treatment with herbal products, like green tea polyphenols, have been proposed $[\underline{3}, \underline{23}, \underline{24}]$. Green tea is a very popular beverage consumed daily worldwide and green tea extracts are often used in different dietary supplements. Very recently, a large cohort study, which included 733 RA patients, demonstrated that subjects consuming higher amount of tea (>750 mL/day) were associated with lower RA disease activity, suggesting the potential beneficial effect of tea [25]. However, in a similar study, based on the Women's Health Initiative Observational Study cohort (185 women self-reporting validated cases of RA observed for 3 years), only a small association between daily caffeinated, non-herbal tea consumption and incident RA was observed [26].

Similar ambiguous results have been reported for EGCG, one of the main catechins in tea extract, that showed beneficial effects in modulating inflammation in animal models of experimental arthritis $[\underline{2}, \underline{27}]$ with multiple positive effects on health. However, EGCG, as other phenolic compounds, can induce both antioxidant and pro-oxidant effects. If the latter can result beneficial in cancer prevention and therapy since they can stimulate endogenous antioxidant systems with the consequent induction of apoptosis in cancerous and precancerous cells $[\underline{28}, \underline{29}]$, the potential harmful effects of pro-oxidant EGCG in inflammatory pathologies, such as RA, remains to be determined.

To investigate if green tea polyphenols could be beneficial per se or ameliorate the therapeutic effect of MTX by improving its efficacy and/or reducing its negative side effects, we decided to study the efficacy of their co-administration in an experimental model of adjuvant arthritis. This animal model shows clinical and pathological features similar to human RA and, although it may not reproduce all the features of the human disease, it resulted helpful in understanding normal inflammatory and immune responses during RA [ $\underline{30}$, 31]. As green tea extract, we employed a standardized and commercially available 
formulation of decaffeinated green tea (GreenSelect@, from Indena, Italy) which contains > $35 \%$ of EGCG equivalents.

In our study, combination of GS and MTX treatment in arthritic rats did not produce negative effects considering clinical and biochemical parameters (hind paw volume, antioxidative and antiinflammatory effect), but the efficacy of MTX was only weakly ameliorated by GS. The time course analysis of basic arthritic parameters (change of body weight and arthritic score) indicates that GS gradually decreased the effectiveness of MTX. Similar effect of high tea consumption has been found in Iraqi patients affected by and treated with MTX. Tea consumption was positively correlated with swelling joint count [32]. This effect can be partially explained evoking the inhibition of EGCG and other tea catechins on PCFT [ $\underline{7}, \underline{33}$, 34]. Shortly, MTX is transported into cells by PCFT with a $\mathrm{K}_{\mathrm{m}}$ of about $5 \mu \mathrm{M}$ and PCFTmediated folic acid uptake is competitively inibited by EGCG $\left(K_{i}=9 \mu \mathrm{M}\right)$. Therefore, it has been suggested that absorption of MTX can be strongly reduced by consumption of green or black tea []]. Other phenolic compounds, such as myricetin and isoxanthohumol showed similar inhibitory effects on folic acid and MTX uptake [34].

Our data are largely in agreement with a previous work that explored the combination therapy of MTX and EGCG in the same rat model of AA [35]. Although the authors measured different markers of antiarthritic effect, antioxidant and anti-inflammatory responses, they concluded that the treatment with EGCG (100 mg/kg/die for 28 days) ameliorated arthritis progression although not at the same extent of MTX and provided pronounced antiinflammatory and immunomodulatory activity against immune suppressive properties of MTX. Roy et al. showed that the combination of EGCG and MTX improved several of the biological parameters investigate compared to the single treatments, although it was not easy to determine if these effects were synergic or only partially additive [35]. Differently than us, the authors commented that administration of MTX and EGCG was more effectively than did 
MTX in suppressing arthritic progression in rats. This discrepancy between the two works can be attributed to the different source of EGCG employed (pure compound vs enriched extract). It is possible that the presence in GS of other catechins and polyphenol families could be responsible for the different responses of the combined treatments. Finally, an important measurement, such as changes in body weight, that could help to better compare the two works was not determined in the Roy et al. paper.

Despite this interference of green tea extract on MTX uptake and effects evidenced in the present work, GS administration in combination with MTX was associated with interesting outcomes in terms of anti-inflammatory response in the rat model of AA. We investigated the effect of GS on IL-17, a pro-inflammatory cytokine secreted by activated memory CD4+ T cells and on MCP-1, a potent chemotactic agent for monocytes/macrophages, both found to be elevated in RA. IL-17 contributes to the inflammation pathogenesis in RA; in fact, studies from osteoarthritis and skin fibroblasts showed that IL-17 enhanced the effect of IL-1 $\beta$ and TNF- $\alpha$ on the production of IL- 6 and IL-8 $[\underline{36}, \underline{37]}$. In parallel, in a mice model of autoimmune arthritis, EGCG attenuated the arthritic symptoms by inhibition of STAT3 and HIF-1 $\alpha$ pathway with Th17/Treg control [38]. In the present work, we demonstrated that GS tended to counteract the increase of IL-17 in AA rats, although its effect was not statically significant, and persisted in the presence of MTX (Fig. 3B). The IL-17 response to GS and MTX was transient since it was present at day 14, but disappeared at day 28. More interesting the results of GS administration on the plasma levels of MCP-1 that were significantly reduced (Fig. 3A). MCP-1 is mainly produced locally by activated cells, where it may exacerbate and sustain inflammation by attracting pro-inflammatory leukocytes, predominantly monocytes [39]. Similarly to our study, in a mouse collagen induced arthritis model, EGCG (20 mg/kg, intraperitoneally) caused a reduction in the amount MCP-1 produced in osteoblasts [40]. It is worthwhile to note that the combined administration of 
GS+MTX was associated with a significant improvement of MCP-1 plasma concentration for the entire length of the treatment, while, at least at day 14 , the single treatments were ineffective in AA rats (Fig. 3A).

Liver has been shown to play a critical role in modulating the immune response in chronic inflammatory diseases, including RA [41]. Expression of iNOS is under control of numerous pro-inflammatory pathways involved in RA pathogenesis: nuclear factor $\kappa \mathrm{B}(\mathrm{NF}-\kappa \mathrm{B})$, mitogen activated protein kinases (MAPKs) and Janus kinases/signal transducers and activators of transcription (JAK/STAT1/3) [는 $\underline{43}]$. EGCG was effective in the inhibition of $\mathrm{NF}-\kappa \mathrm{B}$ activity with the consequent decreased expression of iNOS and reduced formation of NO-generated ROS $[\underline{44}, \underline{45}]$. According to these previous observations, we reported that GS monotreatment in AA rats significantly inhibited iNOS mRNA although in a less extent compared to MTX (Fig. 4A). A very similar trend was observed for GGT enzymatic activity (Fig. 4B). Although it is well established that the main GGT is related to GSH homeostasis and its main function is the cleavage of GSH and GSH-S-conjugates, new roles have been attributed to GGT as a bone-resorbing factor that stimulates osteoclast formation and accumulates at the inflammation region [16]. Neutralizing antibodies against GGT had therapeutic effect on joint destruction in a collagen-induced arthritis in mice [14]. Our results showed that GS significantly reduced the activity of GGT in spleen of AA rats although, also for GGT activity, MTX in monotherapy was more effective than MTX+GS and GS alone. On the other hand, we found no significant changes in GGT activity by administration of GS in joint homogenates (unpublished results).

Since HO-1 is a cytoprotective enzyme activated by various phytochemicals and we previously demonstrated that quercetin was able to restore its decreased expression in lung and joint of AA rats [12], we investigated the ability of GS to increase HO-1 expression in the same tissues. In our model, levels of HO-1 decreased in the lung of AA rats and MTX was 
able to restore the expression of lung HO-1 to control levels (Fig. 4C). However, GS treatment failed to upregulate $\mathrm{HO}-1$ expression in lung tissue, do not showing any protective effect. Moreover, no significant changes were detected in joint (data not shown). This is in contrast with data reported in literature. In fact, in a collagen-induced arthritis model, it was reported that EGCG-fed mice showed in joint homogenates increased levels of HO-1 compared with PBS-fed mice [46]. Beside the different animal model, the reason for this different response may be related to the different doses of tea catechins employed in these studies.

Surprisingly, GS failed to increase the plasma antioxidant power in the animal control group (see HC-GS bar in Fig. 4D), but, on the opposite, GS decreased it. This is in contrast with the effect of other "antioxidant" phenolic compounds, e.g. quercetin, that, in the same experimental model, showed an opposite effect. In fact, quercetin not only increased the plasma antioxidant capacity in control rats, but also rescued to the basal levels the oxidative stress induced in AA animals [12]. This contradictory behavior can be explained evoking the pro-/antioxidant effect of green tea polyphenols mentioned above although several doubts remain. In fact, it is well established that EGCG (as well as other polyphenols) can autooxidize producing hydrogen peroxide and SOD and catalase can abolish some cellular activities of EGCG by inhibiting its auto-oxidation and dimerization [47]. However, it is not always simple to match these observations with the concept that a bland pro-oxidant effect of polyphenols can stimulate the endogenous antioxidant systems protecting normal tissues from more harmful insults, including carcinogens [28]. Of course, key issues regard not only the chemical structures of the different compounds, but also their bioavailability and biotransformation, and, overall, the doses applied and the time of treatment, e.g. high vs low and acute vs chronic treatment. As an example, a recent paper reported that, in the pristaneinduced arthritis rat model, arthritic symptoms were considerably diminished both in the acute 
and in the chronic phase of the disease by long term oral application of EGCG administered at a daily dose comparable to the one employed in the present study (about 100-160/kg) [48]. The therapeutic effect of EGCG were superimposable to the treatment with MTX used as positive control. Unfortunately, that work did not include a combined treatment.

Finally, we cannot exclude (indeed, it is very likely) that the biological activities of green tea extracts reside behind their pro-/antioxidant properties and it is related to alternative molecular mechanisms of action (regulation of signal transduction pathways, transcription factors, DNA methylation, mitochondrial function, autophagy, etc.) (reviewed in [47]).

\section{Conclusions}

It has been mostly proven that green tea polyphenols in monotherapy can exert antiinflammatory and anti-arthritic effects. However, its pharmacodynamic interaction with MTX must be carefully considered before suggesting a combined treatment. Although sufficient dosage of GS to inhibit MTX activity in humans is not determined, RA patients should be informed about possible negative effect of drinking green tea or taking supplements containing green tea extracts during MTX therapy. More research is needed to establish the safe dose of green tea cups per day for patients treated with MTX. 


\section{Acknowledgements}

The experiment was supported by the Slovak grant agency: APVV-15-0308 and was performed in the frame of three SAV-CNR bilateral projects coordinated by Dr. Katarina Bauerova (Slovakia) and Dr. Gian Luigi Russo (Italy) entitled: "In vitro and in vivo models of arthritic processes to study the mechanisms of inflammation and oxidative stress link-up: New perspectives for arthritis therapy", "Phytochemicals in ameliorating rheumatoid arthritis therapy: from preclinical studies to clinical applications" and "Evaluation of Quercetin and Green Tea in combination with Methotrexate for arthritis therapy”. The authors thank Jana Urgošová and Danica Mihalová for technical assistance.

\section{Conflicts of interest}

The authors declare no conflict of interest 


\section{References}

[1] G.S. Hazlewood, C. Barnabe, G. Tomlinson, D. Marshall, D. Devoe, C. Bombardier. Methotrexate monotherapy and methotrexate combination therapy with traditional and biologic disease modifying antirheumatic drugs for rheumatoid arthritis: abridged Cochrane systematic review and network meta-analysis. BMJ 353 (2016) i1777, doi:10.1136/bmj.i1777.

[2] S. Riegsecker, D. Wiczynski, M.J. Kaplan, S. Ahmed. Potential benefits of green tea polyphenol EGCG in the prevention and treatment of vascular inflammation in rheumatoid arthritis. Life Sci 93 (2013) 307-312, doi:10.1016/j.lfs.2013.07.006.

[3] G. de Almeida Goncalves, A.B. de Sa-Nakanishi, M.M. Wendt, J.F. Comar, C.A. Bersani Amado, A. Bracht, R.M. Peralta. Green tea extract improves the oxidative state of the liver and brain in rats with adjuvant-induced arthritis. Food Funct 6 (2015) 27012711, doi:10.1039/c5fo00548e.

[4] R. Singh, N. Akhtar, T.M. Haqqi. Green tea polyphenol epigallocatechin-3-gallate: inflammation and arthritis. [corrected]. Life Sci 86 (2010) 907-918, doi:10.1016/j.lfs.2010.04.013.

[5] Z. Rasheed, A.N. Anbazhagan, N. Akhtar, S. Ramamurthy, F.R. Voss, T.M. Haqqi. Green tea polyphenol epigallocatechin-3-gallate inhibits advanced glycation end product-induced expression of tumor necrosis factor-alpha and matrix metalloproteinase-13 in human chondrocytes. Arthritis Res Ther 11 (2009) R71, doi:10.1186/ar2700.

[6] H.J. Yun, W.H. Yoo, M.K. Han, Y.R. Lee, J.S. Kim, S.I. Lee. Epigallocatechin-3-gallate suppresses TNF-alpha -induced production of MMP-1 and -3 in rheumatoid arthritis synovial fibroblasts. Rheumatol Int 29 (2008) 23-29, doi:10.1007/s00296-008-0597-5. 
[7] M. Kissei, T. Itoh, T. Narawa. Effect of epigallocatechin gallate on drug transport mediated by the proton-coupled folate transporter. Drug Metab Pharmacokinet 29 (2014) 367-372, doi:10.2133/dmpk.dmpk-14-rg-015.

[8] K. Bauerova, A. Acquaviva, S. Ponist, C. Gardi, D. Vecchio, F. Drafi, B. Arezzini, L. Bezakova, V. Kuncirova, D. Mihalova, et al. Markers of inflammation and oxidative stress studied in adjuvant-induced arthritis in the rat on systemic and local level affected by pinosylvin and methotrexate and their combination. Autoimmunity 48 (2015) 4656, doi:10.3109/08916934.2014.939268.

[9] R.A. Isbrucker, J.A. Edwards, E. Wolz, A. Davidovich, J. Bausch. Safety studies on epigallocatechin gallate (EGCG) preparations. Part 2: dermal, acute and short-term toxicity studies. Food Chem Toxicol 44 (2006) 636-650, doi:10.1016/j.fct.2005.11.003.

[10]R.A. Isbrucker, J. Bausch, J.A. Edwards, E. Wolz. Safety studies on epigallocatechin gallate (EGCG) preparations. Part 1: genotoxicity. Food Chem Toxicol 44 (2006) 626635, doi:10.1016/j.fct.2005.07.005.

[11]V. Kuncirova, S. Ponist, D. Mihalova, F. Drafi, R. Nosal, A. Acquaviva, C. Gardi, J. Harmatha, I. Hradkova, K. Bauerova. N-feruloylserotonin in preventive combination therapy with methotrexate reduced inflammation in adjuvant arthritis. Fundam Clin Pharmacol 28 (2014) 616-626, doi:10.1111/fcp.12085.

[12]C. Gardi, K. Bauerova, B. Stringa, V. Kuncirova, L. Slovak, S. Ponist, F. Drafi, L. Bezakova, I. Tedesco, A. Acquaviva, et al. Quercetin reduced inflammation and increased antioxidant defense in rat adjuvant arthritis. Arch Biochem Biophys 583 (2015) 150-157, doi:10.1016/j.abb.2015.08.008.

[13]O. Ondrejickova, A. Ziegelhoeffer, I. Gabauer, R. Sotnikova, J. Styk, P. Gibala, J. Sedlak, L. Horakova. Evaluation of ischemia-reperfusion injury by malondialdehyde, glutathione and gamma-glutamyl transpeptidase: lack of specific local effects in diverse 
parts of the dog heart following acute coronary occlusion. Cardioscience 4 (1993) 225230.

[14]A. Leonidou, P. Lepetsos, M. Mintzas, E. Kenanidis, G. Macheras, M. Tzetis, M. Potoupnis, E. Tsiridis. Inducible nitric oxide synthase as a target for osteoarthritis treatment. Expert Opin Ther Targets $22 \quad$ (2018) 299-318, doi:10.1080/14728222.2018.1448062.

[15]L. Paskova, V. Kuncirova, S. Ponist, D. Mihalova, R. Nosal, J. Harmatha, I. Hradkova, T. Cavojsky, F. Bilka, K. Siskova, et al. Effect of N-Feruloylserotonin and Methotrexate on Severity of Experimental Arthritis and on Messenger RNA Expression of Key Proinflammatory Markers in Liver. J Immunol Res 2016 (2016) 7509653, doi:10.1155/2016/7509653.

[16]Y. Ishizuka, S. Moriwaki, M. Kawahara-Hanaoka, Y. Uemura, I. Serizawa, M. Miyauchi, S. Shibata, T. Kanaya, T. Takata, N. Taniguchi, et al. Treatment with antigamma-glutamyl transpeptidase antibody attenuates osteolysis in collagen-induced arthritis mice. J Bone Miner Res 22 (2007) 1933-1942, doi:10.1359/jbmr.070726.

[17]S.M. Rachapalli, R. Williams, D.A. Walsh, A. Young, P.D. Kiely, E.H. Choy, N. Early Rheumatoid Arthritis. First-line DMARD choice in early rheumatoid arthritis--do prognostic factors play a role? Rheumatology (Oxford) 49 (2010) 1267-1271, doi:10.1093/rheumatology/kep389.

[18]J.A. Martinez Lopez, E. Loza, L. Carmona. Systematic review on the safety of methotrexate in rheumatoid arthritis regarding the reproductive system (fertility, pregnancy, and breastfeeding). Clin Exp Rheumatol 27 (2009) 678-684.

[19]J.S. Smolen, D. Aletaha, K.P. Machold. Therapeutic strategies in early rheumatoid arthritis. Best Pract Res Clin Rheumatol 19 (2005) 163-177, doi:10.1016/j.berh.2004.08.009. 
[20]G.M. Guidelli, T. Barskova, M.G. Brizi, G. Lepri, A. Parma, R. Talarico, L. Cantarini, B. Frediani. One year in review: novelties in the treatment of rheumatoid arthritis. Clin Exp Rheumatol 33 (2015) 102-108.

[21]A. McLean-Tooke, C. Aldridge, S. Waugh, G.P. Spickett, L. Kay. Methotrexate, rheumatoid arthritis and infection risk: what is the evidence? Rheumatology (Oxford) 48 (2009) 867-871, doi:10.1093/rheumatology/kep101.

[22] M.E. Weinblatt. Rheumatoid arthritis in 2003: where are we now with treatment? Ann Rheum Dis 62 Suppl 2 (2003) ii94-96, doi:10.1136/ard.62.suppl_2.ii94.

[23] S. Fechtner, A. Singh, M. Chourasia, S. Ahmed. Molecular insights into the differences in anti-inflammatory activities of green tea catechins on IL-1beta signaling in rheumatoid arthritis synovial fibroblasts. Toxicol Appl Pharmacol 329 (2017) 112-120, doi:10.1016/j.taap.2017.05.016.

[24]G. Ramadan, N.M. El-Beih, R.M. Talaat, E.A. Abd El-Ghffar. Anti-inflammatory activity of green versus black tea aqueous extract in a rat model of human rheumatoid arthritis. Int J Rheum Dis 20 (2017) 203-213, doi:10.1111/1756-185X.12666.

[25]J. Jin, J. Li, Y. Gan, J. Liu, X. Zhao, J. Chen, R. Zhang, Y. Zhong, X. Chen, L. Wu, et al. Tea Consumption Is Associated with Decreased Disease Activity of Rheumatoid Arthritis in a Real-World, Large-Scale Study. Ann Nutr Metab 76 (2020) 54-61, doi:10.1159/000505952.

[26]D. Lamichhane, C. Collins, F. Constantinescu, B. Walitt, M. Pettinger, C. Parks, B.V. Howard. Coffee and Tea Consumption in Relation to Risk of Rheumatoid Arthritis in the Women's Health Initiative Observational Cohort. J Clin Rheumatol 25 (2019) 127132, doi:10.1097/RHU.0000000000000788.

[27]A. Karatas, A.F. Dagli, C. Orhan, H. Gencoglu, M. Ozgen, N. Sahin, K. Sahin, S.S. Koca. Epigallocatechin 3-gallate attenuates arthritis by regulating $\mathrm{Nrf} 2, \mathrm{HO}-1$, and 
cytokine levels in an experimental arthritis model. Biotechnol Appl Biochem 10.1002/bab.1860(2019), doi:10.1002/bab.1860.

[28]J.D. Lambert, R.J. Elias. The antioxidant and pro-oxidant activities of green tea polyphenols: a role in cancer prevention. Arch Biochem Biophys 501 (2010) 65-72, doi:10.1016/j.abb.2010.06.013.

[29] S. Azam, N. Hadi, N.U. Khan, S.M. Hadi. Prooxidant property of green tea polyphenols epicatechin and epigallocatechin-3-gallate: implications for anticancer properties. Toxicol In Vitro 18 (2004) 555-561, doi:10.1016/j.tiv.2003.12.012.

[30]A. Franch, S. Cassany, C. Castellote, M. Castell. Adjuvant arthritis pretreatment with type II collagen and Mycobacterium butyricum. Immunobiology 186 (1992) 351-361, doi:10.1016/s0171-2985(11)80390-6.

[31]B. Joe, R.L. Wilder. Animal models of rheumatoid arthritis. Mol Med Today 5 (1999) 367-369, doi:10.1016/s1357-4310(99)01528-2.

[32]E.M. Mikhael, S.I. Mohammed. The Effect of Methotrexate in Rheumatoid Arthritis Patients is Reduced by Tea Consumption. American Journal of Pharmacological Sciences 1 (2013) 53-55, doi:10.12691/ajps-1-4-2.

[33]E. Navarro-Peran, J. Cabezas-Herrera, F. Garcia-Canovas, M.C. Durrant, R.N. Thorneley, J.N. Rodriguez-Lopez. The antifolate activity of tea catechins. Cancer Res 65 (2005) 2059-2064, doi:10.1158/0008-5472.CAN-04-3469.

[34]C. Lemos, G.J. Peters, G. Jansen, F. Martel, C. Calhau. Modulation of folate uptake in cultured human colon adenocarcinoma Caco-2 cells by dietary compounds. Eur J Nutr 46 (2007) 329-336, doi:10.1007/s00394-007-0670-y.

[35]S. Roy, S. Sannigrahi, R.P. Vaddepalli, B. Ghosh, P. Pusp. A novel combination of methotrexate and epigallocatechin attenuates the overexpression of pro-inflammatory 
cartilage cytokines and modulates antioxidant status in adjuvant arthritic rats. Inflammation 35 (2012) 1435-1447, doi:10.1007/s10753-012-9457-2.

[36] M.L. Cho, Y.O. Jung, K.W. Kim, M.K. Park, H.J. Oh, J.H. Ju, Y.G. Cho, J.K. Min, S.I. Kim, S.H. Park, et al. IL-17 induces the production of IL-16 in rheumatoid arthritis. Exp Mol Med 40 (2008) 237-245, doi:10.3858/emm.2008.40.2.237.

[37]Y. Katz, O. Nadiv, Y. Beer. Interleukin-17 enhances tumor necrosis factor alphainduced synthesis of interleukins 1,6, and 8 in skin and synovial fibroblasts: a possible role as a "fine-tuning cytokine" in inflammation processes. Arthritis Rheum 44 (2001) 2176-2184, doi:10.1002/1529-0131(200109)44:9<2176::aid-art371>3.0.co;2-4.

[38]E.J. Yang, J. Lee, S.Y. Lee, E.K. Kim, Y.M. Moon, Y.O. Jung, S.H. Park, M.L. Cho. EGCG attenuates autoimmune arthritis by inhibition of STAT3 and HIF-1alpha with Th17/Treg control. PLoS One 9 (2014) e86062, doi:10.1371/journal.pone.0086062.

[39] S.L. Deshmane, S. Kremlev, S. Amini, B.E. Sawaya. Monocyte chemoattractant protein-1 (MCP-1): an overview. J Interferon Cytokine Res 29 (2009) 313-326, doi:10.1089/jir.2008.0027.

[40]S.K. Lin, H.H. Chang, Y.J. Chen, C.C. Wang, D.L. Galson, C.Y. Hong, S.H. Kok. Epigallocatechin-3-gallate diminishes CCL2 expression in human osteoblastic cells via up-regulation of phosphatidylinositol 3-Kinase/Akt/Raf-1 interaction: a potential therapeutic benefit for arthritis. Arthritis Rheum 58 (2008) 3145-3156, doi:10.1002/art.23937.

[41]J.F. Comar, A. Babeto de Sa-Nakanishi, A.L. de Oliveira, M. Marques Nogueira Wendt, C.A. Bersani Amado, E.L. Ishii Iwamoto, R.M. Peralta, A. Bracht. Oxidative state of the liver of rats with adjuvant-induced arthritis. Free Radic Biol Med 58 (2013) 144153, doi:10.1016/j.freeradbiomed.2012.12.003. 
[42]P. Dey, V. Panga, S. Raghunathan. A Cytokine Signalling Network for the Regulation of Inducible Nitric Oxide Synthase Expression in Rheumatoid Arthritis. PLoS One 11 (2016) e0161306, doi:10.1371/journal.pone.0161306.

[43] J.S. Smolen, G. Steiner. Therapeutic strategies for rheumatoid arthritis. Nat Rev Drug Discov 2 (2003) 473-488, doi:10.1038/nrd1109.

[44]J.B. Paquay, G.R. Haenen, G. Stender, S.A. Wiseman, L.B. Tijburg, A. Bast. Protection against nitric oxide toxicity by tea. J Agric Food Chem 48 (2000) 5768-5772, doi:10.1021/jf981316h.

[45]G.L. Tipoe, T.M. Leung, M.W. Hung, M.L. Fung. Green tea polyphenols as an antioxidant and anti-inflammatory agent for cardiovascular protection. Cardiovasc Hematol Disord Drug Targets 7 (2007) 135-144, doi:10.2174/187152907780830905.

[46]S.Y. Min, M. Yan, S.B. Kim, S. Ravikumar, S.R. Kwon, K. Vanarsa, H.Y. Kim, L.S. Davis, C. Mohan. Green Tea Epigallocatechin-3-Gallate Suppresses Autoimmune Arthritis Through Indoleamine-2,3-Dioxygenase Expressing Dendritic Cells and the Nuclear Factor, Erythroid 2-Like 2 Antioxidant Pathway. J Inflamm (Lond) 12 (2015) 53, doi:10.1186/s12950-015-0097-9.

[47]H.S. Kim, M.J. Quon, J.A. Kim. New insights into the mechanisms of polyphenols beyond antioxidant properties; lessons from the green tea polyphenol, epigallocatechin 3-gallate. Redox Biol 2 (2014) 187-195, doi:10.1016/j.redox.2013.12.022.

[48]A. Leichsenring, I. Backer, P.G. Furtmuller, C. Obinger, F. Lange, J. Flemmig. LongTerm Effects of (-)-Epigallocatechin Gallate (EGCG) on Pristane-Induced Arthritis (PIA) in Female Dark Agouti Rats. PLoS One 11 (2016) e0152518, doi:10.1371/journal.pone.0152518. 


\section{Figure Legends}

Fig. 1. Effects of GS and MTX (M) in monotherapy and in combination on the progression of AA measured as changes in the body weight. Values are expressed as mean \pm S.E.M. Statistical significance was evaluated applying ANOVA for independent variables: *** $P<0.001$ with respect to $\mathrm{HC} ;+++P<0.001$ with respect to AA group and \#\#\# $P<0.001$ with respect to AA-M group.

Fig. 2. Effects of GS and MTX (M) in monotherapy and in combination on the progression of AA measured as arthritic score. Values are expressed as mean \pm S.E.M. Statistical significance was evaluated applying ANOVA for independent variables: $* * * P<0.001$ with respect to the HC group; $++P<0.01$ and $+++P<0.001$ with respect to the AA group, and \#\#\#P<0.001 with respect to the AA-M group.

Fig. 3. Effects of GS and MTX (M) on plasma MCP-1 (A) and IL-17 (B) concentrations. Values are expressed as mean \pm S.E.M. Statistical significance was evaluated applying ANOVA for independent variables: $* * * P<0.001$ with respect to the HC group; $+++P<$ 0.001 with respect to the AA group and \#\#P $<0.01$ and \# $P<0.05$ with respect to the AA-M group.

Fig. 4. Effect of GS and MTX (M) and their combination on several biochemical markers. (A) Expression of iNOS mRNA in liver homogenates. (B) GGT activity in spleen homogenate. (C) Expression of HO-1 in lung homogenates. The contents of HO-1 were detected on day 28 by immunoblot, as reported in Methods. (D) Effect on plasma antioxidant activity. The total antioxidant capacity in the plasma of treated rats was measured as reported in Methods by 
means of the ABTS assay. Values are expressed as mean \pm S.E.M. Statistical significance was evaluated applying ANOVA for independent variables: $* P<0.05$ and $* * * P<0.001$ with respect to the $\mathrm{HC}$ group; $+P<0.05,++P<0.01$ and $+++P<0.001$ with respect to the AA group. 